\title{
Fine Needle Aspiration Cytology, Do cytology technicians make the difference?
}

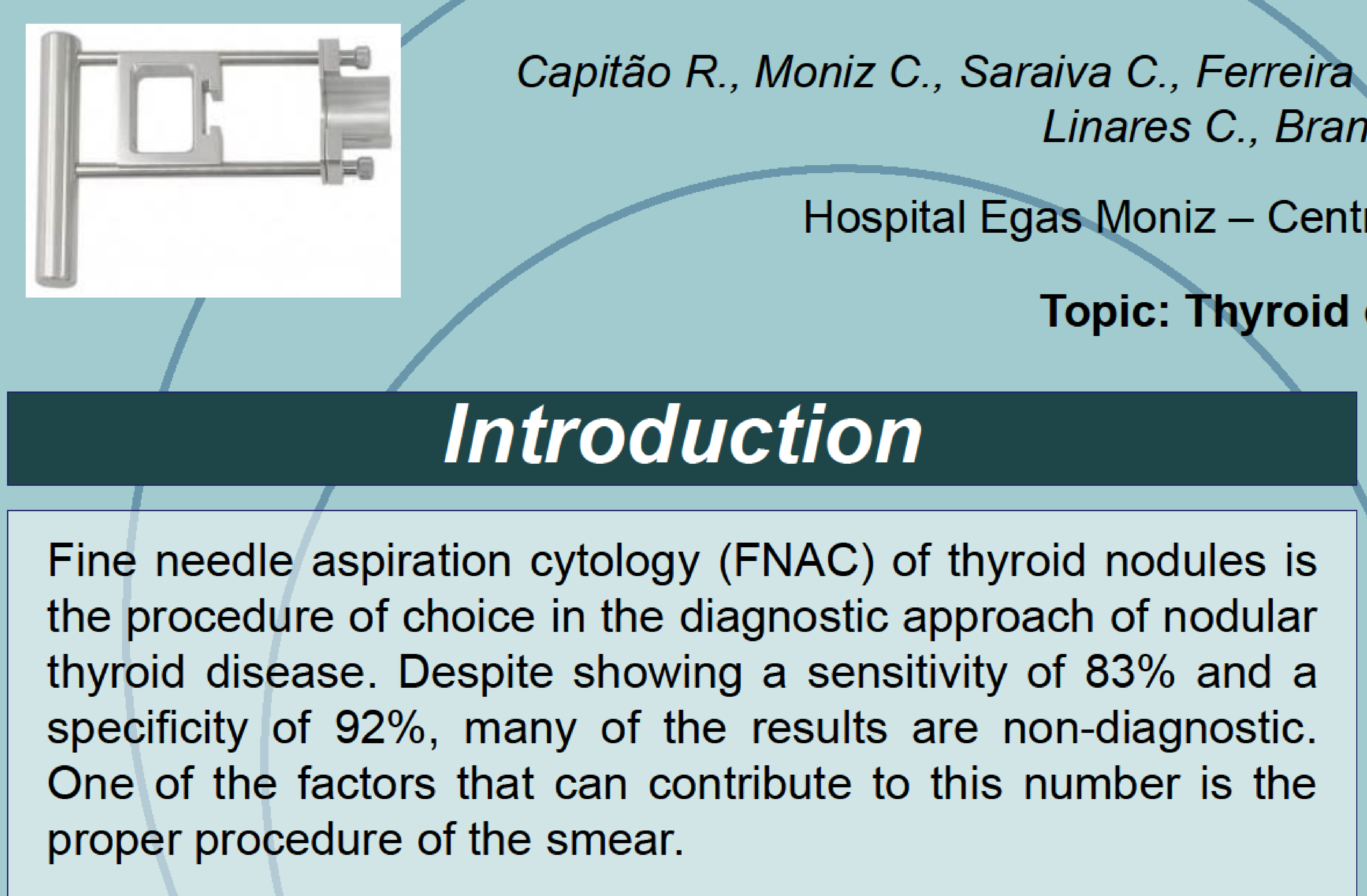

\section{Methods}

All FNACs results were registered in the period of 2 consecutive years in our hospital, according to Bethesda classification. The results were distributed between 2 groups:

- Group A: 01/2013 to 12/2013 without CT support.

- Group B: 01/2014 to 12/2014 with CT support. The results were then analyzed and compared using the PASW statistics and Chisquare test.

\section{Results}

A statistical relationship between the presence of a CT and the Bethesda classification in the evaluation of FNACs was obtained ( $X 2$ test, $p<0.001)$. There were also a significantly more nodules with a non-diagnostic result in the Group $A(X 2$ test, $p<0.001)$ than in Group B (33\% versus $25 \%$ respectively).

The categories II (benign lesions) and IV (follicular tumors) had significantly more results in Group B (59.1\% and $1.5 \%$ ) when compared to Group A (53.0\% and $0.6 \%)$, ( 2 test, $p<0.001$ for category II; $p<0.02$ for category IV). The presence of a CT had an impact on results of FNAC, diminishing the number of non-diagnostic results and increasing the results of benign lesions and follicular tumors.

\begin{tabular}{lccccccc}
\hline Group I Bethesda & I & II & III & IV & V & VI & Total \\
\hline Group A & 510 & 815 & 173 & 9 & 6 & 25 & 1538 \\
Group B & 384 & 928 & 205 & 23 & 12 & 17 & 1569 \\
Total & 894 & 1743 & 378 & 32 & 18 & 42 & 3107 \\
\hline
\end{tabular}

Table 1 - Total results of FNACs sorted by group A and B

\section{Conclusions}

A CT should be integrated in the cytology team, if possible, in order to reduce the number of non-diagnostic results and consequently the number of patients that need to repeat the FNAC.

The reasons that probably contributed to decrease the number of non-diagnostic lesion were the more promptness in the smear preparation, better smear technique, the macroscopic recognition of inadequate samples and better communication between the $\mathrm{CT}$ and the one who performs the FNAC. 